\title{
Allergic Contact Dermatitis to Epoxy Resin: Case Series study of 24 Patients
}

\section{Deepthi Ravi' ${ }^{1}$, Jayakar Thomas*2}

${ }^{1}$ Assistant Professor, Department of Dermatology, Sree Balaji Medical College and Bharath University, Chennai, India. ${ }^{2}$ Professor and Head, Department of Dermatology, Sree Balaji Medical College and Bharath University, Chennai, India. jayakarthomas@gmail.com

*Corresponding Author: Dr. Jayakar Thomas, Professor and Head, Department of Dermatology, Sree Balaji Medical College and Bharath University, Chennai, India.

\section{Abstract}

Epoxy resin systems have strong adhesive, toughness and high mechanical and thermal resistance. They are used in electronics, electrical components, light emitting diodes, electrical insulators, metal coatings, structural adhesives and fiber reinforced plastic materials. Here we report a series of 24 patients presenting with allergic contact dermatitis coming from a factory manufacturing electronic chips containing epoxy resin.

Keywords: Allergic contact dermatitis; epoxy resin; electronic chips.

\section{INTRODUCTION}

The epoxy resin system has wide industrial usage in electric components, electronics, electrical insulators, light emitting diodes and many other materials such as structural adhesives and fiber reinforced plastic materials. Epoxy resins are a part of the epoxy resin system. Uncured epoxy resins have poor chemical, mechanical and heat resistance properties. Curing or hardening of the resin is necessary for obtaining good properties. The most common allergen is diglycidylether of bisphenol $\mathrm{A}^{1}$ but other allergens which can lead to contact dermatitis include epichlorohydrin and diglycidyl ether.An allergic reaction can also develop to the other components of the epoxy resin system such as hardeners and reactive diluents.

\section{CASE REPORT}

24 patients presented to Sree Balaji Medical college from the same factory located in Chennai. The factory manufactures electronic chips. All the patients were female. The age distribution ranged from 21 to 39 . The duration of the lesions ranged from a period of 2 weeks to 3 months.

The patients had history of transporting the chips during which it came in contact with forearms and the hands. The patients were instructed to wear gloves and eyeshields but admitted to not using it regularly. Moreover, some of the patients had only eyelid lesions which may indicate an airborne contact dermatitis to the hardeners.

The patients presented with itchy lesions over the flexor aspect of the forearms, eyelids, front of the neck, dorsa of the hands and abdomen (Figure 1\&2). 10 patients had multiple site involvement. The most common site involved was the forearms (14 patients) followed by the eyelids (5), face (5), hands (4), neck (3) and the abdomen (1).

The lesions ranged from erythema, slightly scaling to hyperpigmented excoriated plaques.

The patients were treated with low to mid-potent topical corticosteroids and anti-histamines. Most of the patients did not return for a follow-up since their factory rules had only one mandatory check-up for the patients. A patch test to confirm the allergen could not be done due to this reason. The chips and work place materials for patch testing also could not be obtained to the factory norms.

\section{Discussion}

The most common type of allergic reaction to epoxy resin is contact dermatitis and it occurs most commonly on contact with uncured epoxy resin. 


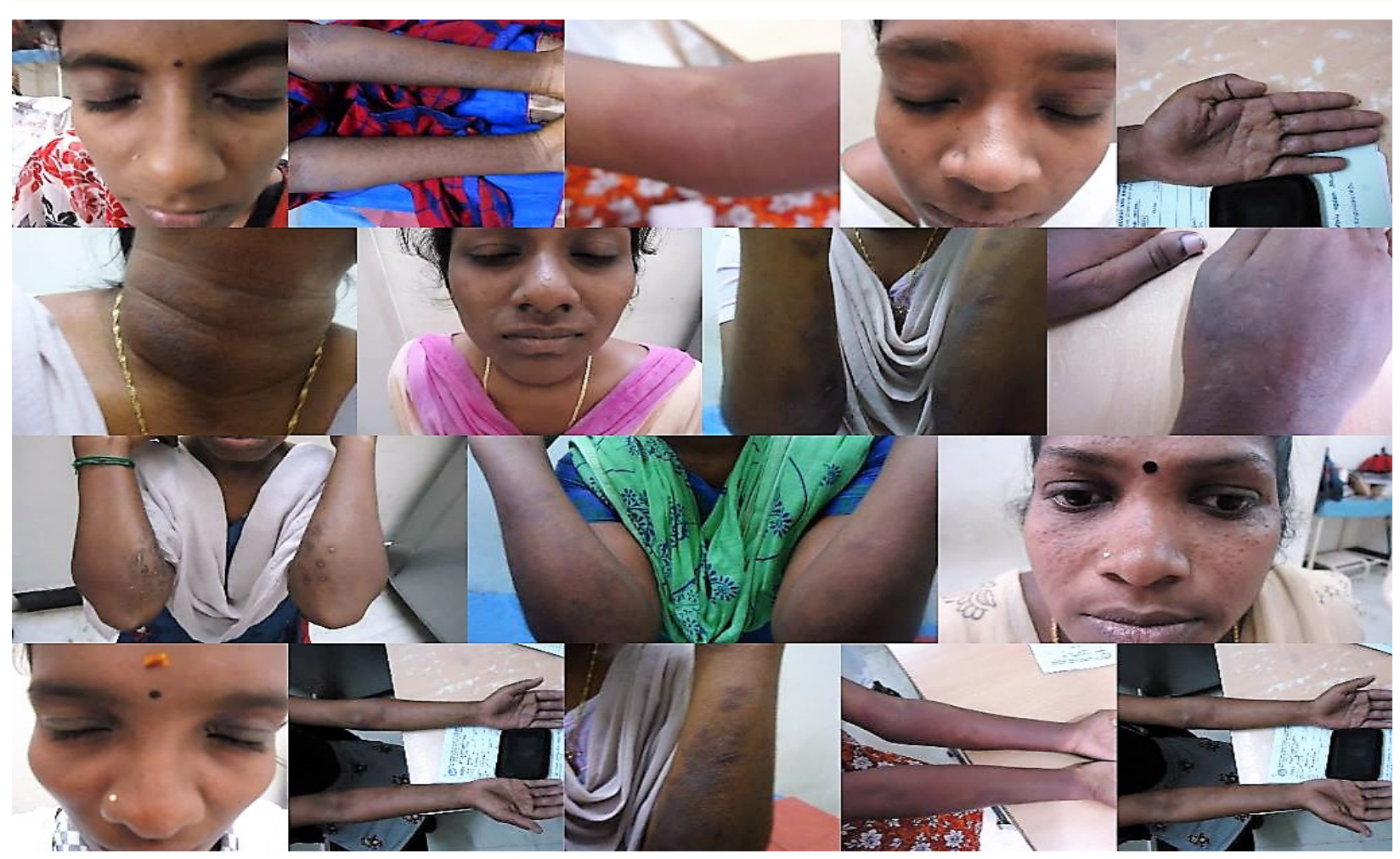

Figure 1.14 patients showing allergic contact dermatitis to epoxy resin. The most common site is the upper extremities especially the forearms

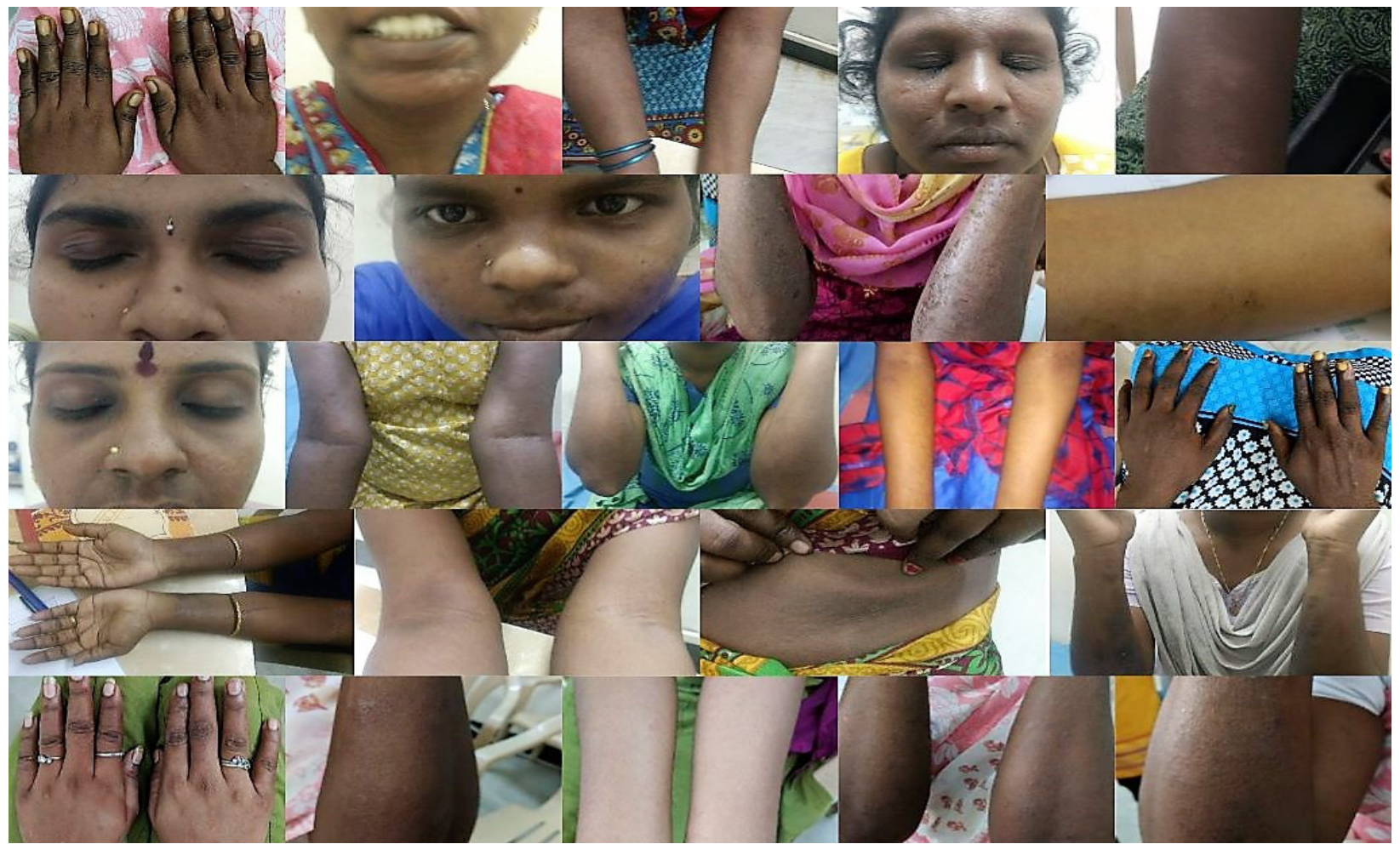

Figure 2. 10 patients showing a reaction to epoxy resin. The eyelids are also involved indicating an airborne contact dermatitis component. The forearms were the areas which commonly came in contact with the chips and hence were most commonly affected. 
However, contact dermatitis to the epoxy hardener isophorone diamine and the reactive diluent phenyl glycidyl ether has also been reported ${ }^{2}$. Hypersensitivity pneumonitis and inhalation fever can also occur due to epoxy fumes or dust. If either of these conditions occur, the patient should strictly avoid epoxy resin exposure.

The most common site affected in the patients studied was the forearms which also corroborates with a study by Kristiina et $\mathrm{al}^{3}$. In the same study, the most common allergen which tested positive in patch testing was diglycidylether of bisphenol A. However, the study came to a conclusion that diagnosis of allergic contact dermatitis to epoxy products need to be tested with workplace products and that testing with commercial test substances is not sufficient.

The patients in our study presented with face especially eyelid lesions. This may be as a result of airborne contact dermatitis due to airborne reactive diluents and hardeners. A similar dermatitis was reported in a case report by Isaksson et al in a golfer presenting with face and hand eczema ${ }^{4}$.

Some of the patients in our study presenting only with eyelid lesions did not come in contact with the chips containing the resin and were involved in office jobs. They may have developedonly airborne contact dermatitis.

Patch testing is necessary to confirm the diagnosis of allergic contact dermatitis to epoxy resin. This was not possible in our study because the patient presented with active lesions and did not report for follow up.

The patients need to be treated with topical emollients and steroids, Avoidance of the resin is advised. Recurrence is common after re-exposure to the resin especially in sensitive individuals.

Since it is difficult for the patients to change jobs, it is important for them to maintain precautions such as use of goggles, protective clothing and nitrile rubber gloves. Moreover, the patients should be instructed to change clothing and take a bath after leaving the work place to reduce the incidence of airborne contact dermatitis.

\section{ConClusion}

A patch test is needed to confirm the diagnosis of allergic contact dermatitis to epoxy resin. Finding allergy to the hardeners and diluents is also important. It is preferable to obtain the allergens from the workplace for testing.

Avoidance of the allergen along with corticosteroids and emollients are recommended for treatment of the condition. Complete avoidance may be difficult unless there is change of workplace which may be logistically difficult for the patient. Hence, Nitrile butyl or nitrile rubber gloves along with protective clothing and goggles is advised.

\section{REFERENCES}

[1] Maria MC,MargaridaG, Americo F. Contact allergy to epoxy resins- A 10-year study. Contact Dermatitis 2010: 62: pg no.55.

[2] Marius R. Occupational epoxy resin allergic contact dermatitis. Australian Journal of Dermatology 2000. 41(4): pg no. 222-224.

[3] Kristiina A.K, Maria P, Katri S. Occupational allergic contact dermatitis caused by epoxy chemicals: occupations, sensitizing products, and diagnosis. Contact Dermatitis. 73: pg no. 336-342.

[4] Isaksson M, Möller H, Pontén A. Occupational allergic contact dermatitis from epoxy resin in a golf club repairman.Dermatitis. 2008;19(5): pg no.30-32.

Citation: Deepthi Ravi, Jayakar Thomas. Allergic Contact Dermatitis to Epoxy Resin: Case Series study of 24 Patients. Archives of Dermatology and Skin Care. 2018; 1(1): 26-28.

Copyright: (C) 2018 Deepthi Ravi, Jayakar Thomas. This is an open access article distributed under the Creative Commons Attribution License, which permits unrestricted use, distribution, and reproduction in any medium, provided the original work is properly cited. 